\title{
Some Estimators for the Pareto Distribution
}

\author{
Gyan Prakash* \\ Department of Statistics, Harish Chandra P. G. College, Varanasi, U. P., India \\ Received 27 November 2008, accepted in final revised form 29 March 2009
}

\begin{abstract}
We derive some shrinkage test-estimators and the Bayes estimators for the shape parameter of a Pareto distribution under the general entropy loss (GEL) function. The properties have been studied in terms of relative efficiency. The choices of shrinkage factor are also suggested.
\end{abstract}

Keywords: General entropy loss; Shrinkage factor; Shrinkage test-estimator; Bayes estimator; Relative efficiency.

(C) 2009 JSR Publications. ISSN: 2070-0237 (Print); 2070-0245 (Online). All rights reserved.

DOI: $10.3329 /$ jsr.v1i2.1642

\section{Introduction}

The Pareto distribution and its close relatives provide very flexible family of fat-tailed distributions which may be used as a model for income distribution of higher income group. Davis and Feldstein [1] have viewed the Pareto distribution as a potential model for the life testing problems. This distribution has established its important role in variety of other problems such as size of cities and firms [2], business mortality [3], service time in queuing system [4].

The Pareto distribution has played a major role in the investigations of previous phenomena providing a satisfactory model at the extremities. It plays an important role in socio-economic studies. It is often used as a model for analysis area including city population distribution, stock price fluctuations and oil field locations. Also, it has application in military areas and suitable for approximating the right tails of distribution with positive skewness. The Pareto law applied to study the distributions of nuclear particles [5]. Harris [6] used this distribution in determining times of maintenance service while Dyer [7] found that the two-parameter Pareto distribution transformation is equivalent to the two-parameter exponential distribution. The probability density function of the classical Pareto distribution is given as

$$
f(x ; \sigma, \theta)=\theta \sigma^{\theta} x^{-(\theta+1)} ; x \geq \sigma, \theta>0 .
$$

*Corresponding author: ggyanji@yahoo.com 
Here, $\theta$ be the shape parameter and $\sigma$ is a scale parameter. Let $x_{1}, x_{2}, \ldots, x_{n}$ be a random sample of size $n$ drawn from the distribution (Eq. 1). The maximum likelihood estimators (MLE) and the unbiased estimators for the parameters $\theta$ and $\sigma$ are given as

$$
\begin{array}{c|c}
\hat{\theta}=\left[\frac{1}{n} \sum_{i=1}^{n} \log \left(\frac{x_{i}}{x_{(1)}}\right)\right]^{-1} & \hat{\sigma}=x_{(1)}=\min \left(x_{1}, x_{2}, \ldots x_{n}\right) \\
\hline \hat{\theta}_{u}=\frac{n-2}{n} \hat{\theta} & \hat{\sigma}_{u}=\left[1-\frac{1}{(n-1) \hat{\theta}}\right] \hat{\sigma}
\end{array}
$$

Here, suffix $u$ stands for unbiased estimator. Also, $2 \theta_{0}(n-2) \theta_{u}^{-1}$ is distributed as a chi-square distribution with $2(n-1)$ degrees of freedom

It is recognized that a shrinkage estimator performs better if a guess value of the parameter is in the vicinity of the true value and the sample size is small. Following Thompson [8], a shrinkage estimator of $\theta$ is

$$
T=k \hat{\theta}_{u}+(1-k) \theta_{0},
$$

where $\theta_{0}$ is a guess value of the parameter $\theta$. The shrinkage factor $k$ lies between 0 and 1 and is specified by the experimenter according to his belief in the guess value $\theta_{0}$. The shrinkage procedure has been applied in a number of problems such as mean survival time in epidemiological studies [9], forecasting of the money supply [10], estimating mortality rates [11] and improved estimation in sample surveys [12]. The performances of the shrinkage estimators utilizing a point guess value has been studied in Refs. [13-18] and others in different contexts.

We know that in many real life situations, the overestimation or underestimation are not of equal consequences. For such situations a useful asymmetric loss function was introduced by Varian [19], called as the LINEX loss function. This function rises approximately exponentially on one side of zero and approximately linearly on the other side. A suitable alternative to the LINEX loss is the general entropy loss (GEL) proposed by Calabria and Pulcini [20] and is given for the parameter $\theta$ as

$$
L(\Delta)=\Delta^{p}-p \log (\Delta)-1 ; p \neq 0, \Delta=\frac{\hat{\theta}_{u}}{\theta} .
$$

The shape parameter $p$ allows different shapes of this loss function. For $p>0$, a positive error $\hat{\theta}_{u}>\theta$ causes more serious consequences than a negative one and vice versa. Also, the minimum occurs at $\hat{\theta}_{u}=\theta$.

In this paper, we propose some shrinkage estimators for the shape parameter $\theta$ when initial estimate of $\theta$ is available in the form of the guess value $\theta_{0}$ with different choices of the shrinkage factor and study their properties in terms of relative efficiency under GEL function. The Bayes estimators of the parameter $\theta$ are also derived when the scale parameter $\sigma$ is known and unknown. 


\section{A Proposed Class of Estimators for the Shape Parameter}

The proposed class of estimators for the shape parameter is given as

$$
Y=C \hat{\theta}_{u} ; C \in R^{+} .
$$

The risk of the estimator $Y$ under GEL (Eq. 3) is obtained as

$$
\begin{aligned}
R(Y)=E & \left\{\left(\frac{C \hat{\theta}_{u}}{\theta}\right)^{p}-p \log \left(\frac{C \hat{\theta}_{u}}{\theta}\right)-1\right\} \\
& =G\left(0, \infty, \Delta^{\prime}\right) ; \Delta^{\prime}=\left(f^{\prime}\right)^{p}-p \log \left(f^{\prime}\right)-1,
\end{aligned}
$$

where $f^{\prime}=C \frac{n-2}{z}, G\left(x_{1}, x_{2}, W\right)=\int_{x_{1}}^{x_{2}} e^{-z}(W) z^{n-2} d z / \int_{0}^{\infty} e^{-z} z^{n-2} d z$ and $W$ may be the function of $z$.

The value of $C$, that minimizes $R(Y)$ is obtained as

$$
C_{1}=\frac{1}{n-2}\left(\frac{\Gamma(n-1)}{\Gamma(n-p-1)}\right)^{1 / p} \text {. }
$$

Hence, the improved estimator among the class (Eq. 4) is

$$
Y_{1}=C_{1} \hat{\theta}_{u}
$$

with the risk

$$
R\left(Y_{1}\right)=G\left(0, \infty, \Delta_{0}\right),
$$

where $\Delta_{0}=\left(f_{0}\right)^{p}-p \log \left(f_{0}\right)-1$ and $f_{0}=\left(\frac{\Gamma(n-1)}{\Gamma(n-p-1)}\right)^{1 / p} \frac{1}{z}$.

\section{The Proposed Shrinkage Estimator and its Properties}

The risk of the estimator $T$ given in Eq. (2) under the GEL is given as

$$
R(T)=G\left(0, \infty, \Delta^{\prime \prime}\right)
$$

where $\Delta^{\prime \prime}=\left(f^{\prime \prime}\right)^{p}-p \log \left(f^{\prime \prime}\right)-1, f^{\prime \prime}=k\left(\frac{n-2}{z}-\delta\right)+\delta$ and $\delta=\frac{\theta_{0}}{\theta}$.

The relative efficiency of the shrinkage estimator $T$ with respect to improved estimator $Y_{1}$ is defined as

$$
R E\left(T, Y_{1}\right)=R\left(Y_{1}\right) / R(T) .
$$

The relative efficiency $R E\left(T, Y_{1}\right)$ is the function of $n, \delta, p$ and k. For the selected values of $n=06,08,10,15 ; \delta=0.25(0.25) 1.75 ; p= \pm 1.00,2.00$ and $k=0.25,0.50,0.75$; the relative efficiencies have been calculated but not presented here. The proposed estimator $T$ is more efficient than improved estimator $Y_{1}$ in the interval $0.75 \leq \delta \leq 1.25$ 
for all parametric values and the effective interval decreases as $n$ increases. Further, the efficiency reaches maximum at the point $\delta=1.00$ and decreases as $n$ increases (except $\delta=1.00$ ). Also, the efficiency first increases for $\delta<1.00$ and then decreases in the interval $\delta>1.00$ as $p$ increases. It is also seen that for the moderate values of $k$ the gain in efficiency is larger in the vicinity of the true value of the parameter, i.e. $(0.75 \leq \delta \leq 1.25)$.

The value of the shrinkage factor $k=k_{1}$ (say), which minimizes $R(T)$ is obtained numerically by solving the given equality

$$
G\left(0, \infty,\left(\frac{n-2}{z}-\delta\right)\left(f^{\prime \prime}\right)^{p-1}\right)=G\left(0, \infty,\left(\frac{n-2}{z}-\delta\right)\left(f^{\prime \prime}\right)^{-1}\right) .
$$

Based on $k_{1}$ the improve shrinkage estimator for $\theta$ is given as

$$
T_{1}=k_{1} \hat{\theta}_{u}+\left(1-k_{1}\right) \theta_{0}
$$

with the risk under the GEL is

$$
R\left(T_{1}\right)=G\left(0, \infty, \Delta_{1}\right),
$$

where $\Delta_{1}=\left(f_{1}\right)^{p}-p \log \left(f_{1}\right)-1$ and $f_{1}=k_{1}\left(\frac{n-2}{z}-\delta\right)+\delta$.

The relative efficiency of $T_{1}$ with respect to $Y_{1}$ is given as

$$
R E\left(T_{1}, Y_{1}\right)=R\left(Y_{1}\right) / R\left(T_{1}\right) .
$$

The relative efficiency $\operatorname{RE}\left(T_{1}, Y_{1}\right)$ is the function of $n, \delta$ and $p$. For the similar set of values as considered earlier, the relative efficiencies have been calculated but not presented here. The improved shrinkage estimator $T_{1}$ is more efficient than $Y_{1}$ in the interval $0.75 \leq \delta \leq 1.50$ for all parametric space and the effective interval decreases as $n$ increases. Other properties are similar to the shrinkage estimator $T$.

\section{The Proposed Shrinkage Test-Estimator and its Properties}

From the above conclusion, we conclude that the shrinkage estimator performs well when guess value is approximately near to the parameter and for the moderate values of sample size as well as the shrinkage factor. This suggests that one may employ a test under the hypothesis $H_{0}: \theta=\theta_{0}$ against $H_{1}: \theta \neq \theta_{0}$. A test statistic $2 \theta_{0}(n-2) \theta_{u}^{-1} \sim \chi_{2(n-1)}^{2} \quad$ is available for testing the hypothesis $\mathrm{H}_{0}$ against $\mathrm{H}_{1}$. Therefore, the proposed shrinkage test-estimator is

$$
\hat{\theta}_{u S H}= \begin{cases}k\left(\hat{\theta}_{u}-\hat{\theta}_{0}\right)+\theta_{0} & \text { if } t_{1} \leq \hat{\theta}_{\mathrm{u}} \leq t_{2} \\ C_{1} \hat{\theta}_{u} & \text { else }\end{cases}
$$

where $t_{1}=\frac{2 \theta_{0}(n-2)}{r_{2}}, t_{2}=\frac{2 \theta_{0}(n-2)}{r_{1}}$, with $r_{1}$ and $r_{2}$ being the values of the lower and upper $100 \alpha / 2 \%$ points of the chi-square distribution with $2(n-1)$ degrees of freedom. 
The relative bias of the shrinkage test-estimator $\hat{\theta}_{u S H}$ is obtained as

$$
R B\left(\hat{\theta}_{u S H}\right)=\frac{E\left(\hat{\theta}_{u S H}\right)}{\theta}-1=G\left(z_{1}, z_{2}, \bar{\Delta}_{0}\right)+C_{1}-1 ; \bar{\Delta}_{0}=\left(f^{\prime \prime}-f_{0}\right) .
$$

The risk under the GEL for the proposed shrinkage test-estimator $\hat{\theta}_{u S H}$ is obtained as

$$
R\left(\hat{\theta}_{u S H}\right)=G\left(z_{1}, z_{2}, \Delta^{\prime \prime}\right)+G\left(0, \infty, \Delta_{0}\right)-G\left(z_{1}, z_{2}, \Delta_{0}\right)
$$

where $z_{1}=\frac{r_{1}}{2 \delta}$ and $z_{2}=\frac{r_{2}}{2 \delta}$.

The relative efficiency of the shrinkage test-estimator $\hat{\theta}_{u S H}$ with respect to $Y_{1}$ is

$$
R E\left(\hat{\theta}_{u S H}, Y_{1}\right)=R\left(Y_{1}\right) / R\left(\hat{\theta}_{u S H}\right) .
$$

The relative bias and the relative efficiency are functions of $n, \delta, p, k$ and $\alpha$ (level of significance). For selected values of $n=06,08,10,15 ; \delta=0.25(0.25) 1.75$; $p= \pm 1.00,2.00 ; k=0.25,0.50,0.75$ and $\alpha=0.01,0.05$; the relative biases and the relative efficiencies have been calculated but not presented here.

The relative bias lies between -0.178 and 0.097 and has a tendency of being positive for $\delta \geq 1.00$ and negative otherwise. The relative biases decrease as $n$ increases for the all considered values of $\delta$. Similar trend has been seen in the interval $0.75 \leq \delta \leq 1.75$ when $\alpha$ increases. Further, the relative bias decreases when $p$ increases for $\delta \geq 1.00$.

The shrinkage test-estimator $\hat{\theta}_{u S H}$ has smaller risk than the minimum risk estimator $Y_{1}$ provided $n$ is small, $k$ is small and in the interval $0.50 \leq \delta \leq 1.50$ and the effective interval decreases with $k$ as well as $n$ increases. The relative efficiency is higher at the point $\delta=1.00$ and it decreases for $n$ as well as $\alpha$ increases when $\delta \leq 1.25$. In addition, the efficiency first increases for $\delta<1.00$ and then decreases in the interval $\delta>1.00$ as $p$ increases. It is also noted that for the moderate values of $k$ the gain in efficiency is larger in the vicinity of the true value and guess value.

\section{Choices of the Shrinkage Factor}

The shrinkage test-estimator based on the shrinkage factor $k_{1}$ (Eq. 9) that minimizes $R(T)$, is given as

$$
\hat{\theta}_{u S H 1}=\left\{\begin{array}{ll}
k_{1}\left(\hat{\theta}_{u}-\hat{\theta}_{0}\right)+\theta_{0} & \text { if } t_{1} \leq \hat{\theta}_{\mathrm{u}} \leq t_{2} \\
C_{1} \hat{\theta}_{u} & \text { else }
\end{array} .\right.
$$

Again, the value of the shrinkage factor $k=k_{2}$ (say), which minimizes the risk of the shrinkage estimator $\hat{\theta}_{u S H}$ given in Eq. (13), is obtained numerically by solving the given equality 


$$
G\left(z_{1}, z_{2},\left(f^{\prime \prime}\right)^{p-1}\right)=G\left(z_{1}, z_{2},\left(f^{\prime \prime}\right)^{-1}\right) .
$$

Based on $k_{2}$ the proposed shrinkage test-estimator is

$$
\hat{\theta}_{u S H 2}=\left\{\begin{array}{ll}
k_{2}\left(\hat{\theta}_{u}-\hat{\theta}_{0}\right)+\theta_{0} & \text { if } t_{1} \leq \hat{\theta}_{\mathrm{u}} \leq t_{2} \\
C_{1} \hat{\theta}_{u} & \text { else }
\end{array} .\right.
$$

The relative bias for the shrinkage test-estimator $\hat{\theta}_{u S H} ; i=1,2$ is obtained as

$$
R B\left(\hat{\theta}_{u S H}\right)=G\left(z_{1}, z_{2}, \bar{\Delta}_{i}\right)+C_{1}-1,
$$

where $\bar{\Delta}_{i}=\left(f_{i}-f_{0}\right), f_{i}=k_{i}\left(\frac{n-2}{z}-\delta\right)+\delta$ and $i=1,2$.

The risk for the proposed shrinkage test-estimator $\hat{\theta}_{u S H i} ; i=1,2$ under the GEL is given as

$$
R\left(\hat{\theta}_{u S H i}\right)=G\left(z_{1}, z_{2}, \Delta_{i}\right)+G\left(0, \infty, \Delta_{0}\right)-G\left(z_{1}, z_{2}, \Delta_{0}\right),
$$

where $\Delta_{i}=\left(f_{i}\right)^{p}-p \log \left(f_{i}\right)-1$.

The relative efficiency for the shrinkage test-estimator $\hat{\theta}_{u S H i} ; i=1,2$ with respect to $Y_{1}$ is

$$
R E\left(\hat{\theta}_{u S H i}, Y_{1}\right)=R\left(Y_{1}\right) / R\left(\hat{\theta}_{u S H i}\right) ; i=1,2 .
$$

The expressions of $R B\left(\hat{\theta}_{u S H i}\right)$ and $R E\left(\hat{\theta}_{u S H i}, Y_{1}\right) ; i=1,2$ are the function of $n$, $\delta, p$ and $\alpha$. For the similar set of selected values as considered earlier the relative biases (not presented here) and the relative efficiencies have been calculated (Tables 1 and 2).

The values of the relative biases are smaller and have a tendency of being positive for $\delta \geq 1.00$ and negative otherwise. The relative biases decrease as $n$ increases for the all considered values of $\delta$. Similar trend has been seen in the interval $0.75 \leq \delta \leq 1.75$ when $\alpha$ increases. Further, the relative bias decreases when $p$ increases for $\delta \geq 1.00$. The relative biases lie between

(1) -0.238 and 0.125 for the test-estimator $\hat{\theta}_{u S H}$

(2) -0.202 and 0.082 for the test-estimator $\hat{\theta}_{u S H} 2$

From Table 1, we conclude that the shrinkage test-estimator $\hat{\theta}_{u S H 1}$ is more efficient than $Y_{1}$ in the interval $0.50 \leq \delta \leq 1.75$ and the efficiency is maximum at the point $\delta=1.00$. The relative efficiency decreases in $\delta \leq 1.25$ when sample size $n$ as well as level of significance $\alpha$ increases. Further, the efficiency first increases in the interval $\delta \leq 0.75$ and then decreases for $\delta \geq 1.25$ when $p$ increases. 
The test-estimator $\hat{\theta}_{u S H_{2}}$ is more efficient than $Y_{1}$ for the all considered values of parametric space and attains maximum efficiency at point $\delta=1.00$ (Table 2). The efficiency decreases in $\delta \leq 1.25$ when $\alpha$ increases. Similar trend has been seen when $p$ increases in the interval $\delta \geq 1.25$.

Table 1. Relative efficiency between shrinkage test-estimator $\hat{\theta}_{u S H 1}$ and $Y_{1}$.

\begin{tabular}{|c|c|c|c|c|c|c|c|c|c|}
\hline & & & & & & $\delta$ & & & \\
\hline$n$ & $\alpha$ & $p$ & 0.25 & 0.50 & 0.75 & 1.00 & 1.25 & 1.50 & 1.75 \\
\hline \multirow{6}{*}{6} & \multirow{3}{*}{0.01} & -1.00 & 0.2459 & 1.9168 & 4.3238 & 7.8084 & 3.6982 & 1.8775 & 1.3782 \\
\hline & & 1.00 & 0.5184 & 2.4774 & 5.8585 & 9.7923 & 3.4937 & 1.7502 & 1.1669 \\
\hline & & 2.00 & 0.7075 & 2.8312 & 6.5902 & 10.786 & 3.3005 & 1.7418 & 1.0976 \\
\hline & \multirow{3}{*}{0.05} & -1.00 & 0.2455 & 1.8806 & 3.0648 & 4.2562 & 2.8659 & 2.0683 & 1.7011 \\
\hline & & 1.00 & 0.4971 & 2.3505 & 3.6909 & 4.1449 & 2.5461 & 1.7355 & 1.3701 \\
\hline & & 2.00 & 0.6611 & 2.5945 & 3.8385 & 4.0858 & 2.4672 & 1.6263 & 1.2382 \\
\hline \multirow{6}{*}{8} & \multirow{3}{*}{0.01} & -1.00 & 0.1762 & 1.6501 & 3.0553 & 5.6100 & 2.6474 & 1.6183 & 1.2787 \\
\hline & & 1.00 & 0.3535 & 2.0093 & 3.9689 & 6.3451 & 2.5695 & 1.4105 & 1.0340 \\
\hline & & 2.00 & 0.4732 & 2.2360 & 4.5107 & 6.9067 & 2.5366 & 1.3415 & 1.0031 \\
\hline & \multirow{3}{*}{0.05} & -1.00 & 0.1751 & 1.6339 & 2.3438 & 3.6902 & 2.6332 & 1.9699 & 1.6693 \\
\hline & & 1.00 & 0.3463 & 1.9608 & 2.7837 & 3.3988 & 2.2500 & 1.6483 & 1.3887 \\
\hline & & 2.00 & 0.4555 & 2.1485 & 3.0103 & 3.3602 & 2.1298 & 1.5116 & 1.2429 \\
\hline \multirow{6}{*}{10} & \multirow{3}{*}{0.01} & -1.00 & 0.1379 & 1.5035 & 2.3623 & 4.5180 & 2.5484 & 1.5465 & 1.2960 \\
\hline & & 1.00 & 0.2678 & 1.7646 & 2.9736 & 4.7424 & 2.2742 & 1.3123 & 1.0477 \\
\hline & & 2.00 & 0.3545 & 1.9258 & 3.3383 & 4.9857 & 2.1957 & 1.2184 & 1.0023 \\
\hline & \multirow{3}{*}{0.05} & -1.00 & 0.1362 & 1.4950 & 1.9276 & 3.4317 & 2.3234 & 1.9347 & 1.6586 \\
\hline & & 1.00 & 0.2654 & 1.7406 & 2.2343 & 3.0317 & 2.1365 & 1.6806 & 1.4708 \\
\hline & & 2.00 & 0.3467 & 1.8847 & 2.4111 & 2.9327 & 2.0263 & 1.5442 & 1.3440 \\
\hline \multirow{6}{*}{15} & \multirow{3}{*}{0.01} & -1.00 & 0.0962 & 1.3219 & 1.5260 & 3.4449 & 2.4545 & 1.6042 & 1.4354 \\
\hline & & 1.00 & 0.1687 & 1.4754 & 1.8305 & 3.2390 & 2.1900 & 1.4057 & 1.2760 \\
\hline & & 2.00 & 0.2175 & 1.5668 & 2.0062 & 3.2072 & 2.1751 & 1.2995 & 1.1727 \\
\hline & \multirow{3}{*}{0.05} & -1.00 & 0.0878 & 1.3203 & 1.4181 & 3.2406 & 2.3169 & 1.7853 & 1.5575 \\
\hline & & 1.00 & 0.1678 & 1.4692 & 1.5754 & 2.7851 & 2.0952 & 1.7447 & 1.5485 \\
\hline & & 2.00 & 0.2173 & 1.5564 & 1.6649 & 2.6090 & 2.0121 & 1.6947 & 1.5239 \\
\hline
\end{tabular}


Table 2. Relative efficiency between shrinkage test-estimator $\hat{\theta}_{u \text { SH } 2}$ and $Y_{1}$.

\begin{tabular}{|c|c|c|c|c|c|c|c|c|c|}
\hline & & & & & & $\delta$ & & & \\
\hline$n$ & $\alpha$ & $p$ & 0.25 & 0.50 & 0.75 & 1.00 & 1.25 & 1.50 & 1.75 \\
\hline \multirow{6}{*}{6} & \multirow{3}{*}{0.01} & -1.00 & 1.0944 & 1.2635 & 1.7020 & 4.7150 & 2.6626 & 1.9626 & 1.9796 \\
\hline & & 1.00 & 1.5524 & 2.1211 & 6.4073 & 8.3159 & 2.6537 & 1.8558 & 1.4505 \\
\hline & & 2.00 & 1.1846 & 1.6529 & 4.0796 & 9.0526 & 2.4357 & 1.2327 & 1.2222 \\
\hline & \multirow{3}{*}{0.05} & -1.00 & 1.0823 & 1.1943 & 1.6273 & 3.3398 & 2.3865 & 2.0596 & 1.9754 \\
\hline & & 1.00 & 1.4274 & 2.0689 & 1.4303 & 3.9871 & 2.1844 & 1.7678 & 1.4225 \\
\hline & & 2.00 & 1.1509 & 1.5952 & 3.7983 & 3.8496 & 2.1108 & 1.6514 & 1.2850 \\
\hline \multirow{6}{*}{8} & \multirow{3}{*}{0.01} & -1.00 & 1.0886 & 1.9325 & 3.5734 & 5.1377 & 3.3584 & 1.9404 & 1.8371 \\
\hline & & 1.00 & 1.4149 & 2.0266 & 2.6147 & 4.6479 & 2.5613 & 1.5072 & 1.1231 \\
\hline & & 2.00 & 1.5576 & 1.7689 & 4.3863 & 6.2233 & 1.5344 & 1.4363 & 1.0200 \\
\hline & \multirow{3}{*}{0.05} & -1.00 & 1.0827 & 1.2994 & 2.7123 & 3.5141 & 2.2024 & 1.8867 & 1.6966 \\
\hline & & 1.00 & 1.2456 & 1.8653 & 2.3273 & 3.2490 & 2.1908 & 1.6526 & 1.4130 \\
\hline & & 2.00 & 1.5233 & 1.6801 & 1.9040 & 2.0697 & 1.5182 & 1.5127 & 1.2648 \\
\hline \multirow{6}{*}{10} & \multirow{3}{*}{0.01} & -1.00 & 1.0273 & 1.7375 & 2.8714 & 4.2383 & 2.2207 & 1.8223 & 1.7273 \\
\hline & & 1.00 & 1.2628 & 1.7876 & 3.6031 & 4.4373 & 2.1873 & 1.3808 & 1.1162 \\
\hline & & 2.00 & 1.5594 & 1.8432 & 2.3664 & 4.6511 & 2.1113 & 1.2839 & 1.0985 \\
\hline & \multirow{3}{*}{0.05} & -1.00 & 1.0224 & 1.7196 & 2.3208 & 3.2945 & 2.2147 & 1.7529 & 1.6603 \\
\hline & & 1.00 & 1.0693 & 1.6040 & 2.6512 & 2.9231 & 2.0955 & 1.6593 & 1.4710 \\
\hline & & 2.00 & 1.4938 & 1.8304 & 2.2487 & 2.3258 & 1.9509 & 1.5217 & 1.3431 \\
\hline \multirow{6}{*}{15} & \multirow{3}{*}{0.01} & -1.00 & 1.1482 & 1.4834 & 1.9528 & 3.3120 & 2.3257 & 1.6218 & 1.4945 \\
\hline & & 1.00 & 1.3947 & 1.4991 & 2.4596 & 3.1202 & 1.8708 & 1.4227 & 1.3000 \\
\hline & & 2.00 & 1.4725 & 1.8038 & 2.4433 & 2.6834 & 1.7652 & 1.3156 & 1.1978 \\
\hline & \multirow{3}{*}{0.05} & -1.00 & 1.1477 & 1.4799 & 1.8223 & 3.1335 & 2.2516 & 1.7463 & 1.5433 \\
\hline & & 1.00 & 1.3872 & 1.4636 & 2.0391 & 2.7170 & 1.7787 & 1.6940 & 1.5265 \\
\hline & & 2.00 & 1.4233 & 1.4224 & 2.3319 & 2.5904 & 1.6678 & 1.6381 & 1.4967 \\
\hline
\end{tabular}

\section{The Bayes Estimator and its Properties}

\section{Case 1: When $\sigma$ is known}

The conjugate prior for the parameter $\theta$ can be taken as two parameters Gamma distribution, having probability density function 


$$
g(\theta)=\frac{b^{a}}{\Gamma(a)} \theta^{a-1} e^{-b \theta} ; \theta>0, a, b>0
$$

and the posterior density will be

$$
g_{1}(\theta)=\frac{(b+U)^{n+a}}{\Gamma(n+a)} \theta^{n+a-1} e^{-(b+U) \theta} ; U=\sum_{i=1}^{n} \log \left(\frac{x_{i}}{\sigma}\right)
$$

The posterior density is again a two parameter Gamma density with parameters $(b+U)$ and $(n+a)$. Here $U$ is a sufficient statistic for the parameter $\theta$ and $2 \theta U \sim$ $\chi_{2 n}^{2}$. The Bayes estimator for the parameter $\theta$ under the GEL is obtained as

$$
\hat{\theta}_{B E}=\left\{E\left(\theta^{-p}\right)\right\}^{-1 / p}=\frac{b^{\prime}}{b+U} ; b^{\prime}=\left(\frac{\Gamma(n+a)}{\Gamma(n+a-p)}\right)^{1 / p}
$$

In particular for $p=-1$, the Bayes estimator under GEL is equal to the posterior mean and is given by

$$
\hat{\theta}_{B S}=\frac{n+a}{b+U} .
$$

The risk of these estimators under the GEL is given by

\begin{tabular}{c|c} 
Estimator & Risk \\
\hline$\hat{\theta}_{B S}$ & $R\left(\hat{\theta}_{B S}\right)=I\left(0, \infty, \Delta_{B 0}\right) ; \Delta_{B 0}=\left(\frac{n+a}{b \theta+y}\right)^{p}-p \log \left(\frac{n+a}{b \theta+y}\right)-1$ \\
\hline$\hat{\theta}_{B E}$ & $R\left(\hat{\theta}_{B E}\right)=I\left(0, \infty, \Delta_{B 1}\right) ; \Delta_{B 1}=\left(\frac{b^{\prime}}{b \theta+y}\right)^{p}-p \log \left(\frac{b^{\prime}}{b \theta+y}\right)-1$
\end{tabular}

where $I(0, \infty, W)=\int_{0}^{\infty} e^{-y}(W) y^{n-1} d y / \int_{0}^{\infty} e^{-y} y^{n-1} d y$ and $W$ may be the function of $y$.

The relative efficiency for the Bayes estimator $\hat{\theta}_{B E}$ with respect to $\hat{\theta}_{B S}$ is

$$
R E\left(\hat{\theta}_{B E}, \hat{\theta}_{B S}\right)=R\left(\hat{\theta}_{B S}\right) / R\left(\hat{\theta}_{B E}\right) .
$$

The relative efficiency $R E\left(\hat{\theta}_{B E}, \hat{\theta}_{B S}\right)$ involved $n, a, b, p$ and $\theta$. For $n=06,08,10,15$; $a=1.00,2.00,4.00,8.00,10,15 ; \quad b=0.50,1.00,2.00 ; \quad p=1.00,2.00$ and $\theta=0.25,0.50(0.50)$ 3.00 the relative efficiencies have been calculated and presented in Table 3 for $b=0.50$ and $p=2.00$ only. 
Table 3. Relative efficiency between shrinkage estimator $\hat{\theta}_{B E}$ and $\hat{\theta}_{B S}$.

\begin{tabular}{|c|c|c|c|c|c|c|c|}
\hline \multicolumn{2}{|c|}{$p=2.00 b=0.50$} & \multicolumn{6}{|c|}{$a$} \\
\hline$n$ & $\theta$ & 1.00 & 2.00 & 4.00 & 8.00 & 10.00 & 15.00 \\
\hline \multirow{7}{*}{06} & 0.25 & 1.8680 & 1.8967 & 1.6389 & 1.3508 & 1.2828 & 1.1896 \\
\hline & 0.50 & 1.8213 & 1.9113 & 1.6604 & 1.3583 & 1.2877 & 1.1918 \\
\hline & 1.00 & 1.6831 & 1.9199 & 1.7035 & 1.3737 & 1.2976 & 1.1960 \\
\hline & 1.50 & 1.4971 & 1.8933 & 1.7471 & 1.3897 & 1.3078 & 1.2003 \\
\hline & 2.00 & 1.2876 & 1.8228 & 1.7902 & 1.4065 & 1.3184 & 1.2046 \\
\hline & 2.50 & 1.1082 & 1.7045 & 1.8313 & 1.4243 & 1.3296 & 1.2092 \\
\hline & 3.00 & 1.0016 & 1.5436 & 1.8676 & 1.4431 & 1.3413 & 1.2138 \\
\hline \multirow{7}{*}{08} & 0.25 & 1.6359 & 1.7253 & 1.5831 & 1.3406 & 1.2768 & 1.1873 \\
\hline & 0.50 & 1.5977 & 1.7255 & 1.5975 & 1.3470 & 1.2811 & 1.1893 \\
\hline & 1.00 & 1.4998 & 1.7126 & 1.6259 & 1.3603 & 1.2900 & 1.1933 \\
\hline & 1.50 & 1.3781 & 1.6781 & 1.6534 & 1.3742 & 1.2993 & 1.1974 \\
\hline & 2.00 & 1.2419 & 1.6187 & 1.6790 & 1.3887 & 1.3089 & 1.2016 \\
\hline & 2.50 & 1.1029 & 1.5335 & 1.7012 & 1.4040 & 1.3190 & 1.2059 \\
\hline & 3.00 & 1.0011 & 1.4250 & 1.7180 & 1.4201 & 1.3296 & 1.2104 \\
\hline \multirow{7}{*}{10} & 0.25 & 1.5029 & 1.6107 & 1.5378 & 1.3322 & 1.2722 & 1.1904 \\
\hline & 0.50 & 1.4708 & 1.6052 & 1.5477 & 1.3378 & 1.2761 & 1.1924 \\
\hline & 1.00 & 1.3941 & 1.5845 & 1.5669 & 1.3495 & 1.2842 & 1.1963 \\
\hline & 1.50 & 1.3033 & 1.5490 & 1.5845 & 1.3617 & 1.2927 & 1.2003 \\
\hline & 2.00 & 1.2033 & 1.4975 & 1.5996 & 1.3744 & 1.3015 & 1.2045 \\
\hline & 2.50 & 1.1002 & 1.4297 & 1.6109 & 1.3877 & 1.3107 & 1.2088 \\
\hline & 3.00 & 1.0006 & 1.3474 & 1.6171 & 1.4016 & 1.3203 & 1.2132 \\
\hline \multirow{7}{*}{15} & 0.25 & 1.3310 & 1.4390 & 1.4511 & 1.3147 & 1.2705 & 1.3344 \\
\hline & 0.50 & 1.3083 & 1.4296 & 1.4551 & 1.3191 & 1.2738 & 1.3377 \\
\hline & 1.00 & 1.2580 & 1.4056 & 1.4619 & 1.3280 & 1.2808 & 1.3446 \\
\hline & 1.50 & 1.2021 & 1.3742 & 1.4666 & 1.3371 & 1.2880 & 1.3518 \\
\hline & 2.00 & 1.1422 & 1.3355 & 1.4687 & 1.3466 & 1.2954 & 1.3591 \\
\hline & 2.50 & 1.0805 & 1.2897 & 1.4674 & 1.3563 & 1.3031 & 1.3668 \\
\hline & 3.00 & 1.0003 & 1.2377 & 1.4621 & 1.3663 & 1.3111 & 1.3747 \\
\hline
\end{tabular}

The Bayes estimator $\hat{\theta}_{B E}$ performs uniformly well with respect to $\hat{\theta}_{B S}$ for the all considered values of the parametric space and the efficiency increases as $p$ increases. Opposite trend is seen when ' $a$ ' increases (for larger values of $a \geq 4.00$ ). The efficiency also decreases when sample size $n$ increases for $a \leq 10.00$. 


\section{Case 2: When $\sigma$ is unknown}

When both parameters are unknown, the joint sufficient statistic for $\theta$ and $\sigma$ are

$$
V=\sum_{i=1}^{n} \log x_{i} \text { and } x_{(1)}=\min \left(x_{1}, x_{2}, \ldots, x_{n}\right) .
$$

The joint prior for $\theta$ and $\sigma$ as the product of their respective prior and defined as

$$
f(\sigma, \theta)=\left(\varepsilon \sigma^{\varepsilon-1} \sigma_{0}^{-\varepsilon}\right)\left(\frac{b^{a}}{\Gamma(a)} \theta^{a-1} e^{-b \theta}\right) ; \theta, a, b, \varepsilon>0, \sigma<\sigma_{0}
$$

and the joint posterior density will be

$$
f_{1}(\theta, \sigma) \propto \sigma^{n \theta+\varepsilon-1} \theta^{n+a-1} e^{-(b+V) \theta} ; \theta>0, \sigma<\min \left(\rho, x_{(1)}\right) .
$$

Now, the marginal posterior density of $\theta$ is

$$
f_{2}(\theta)=\frac{1}{J\left(s_{1}, s_{2}, s_{3}\right)}\left(\theta+s_{2}\right)^{-1} \theta^{s_{1}-1} e^{-s_{3} \theta} ; \theta>0
$$

where $\quad J\left(s_{1}, s_{2}, s_{3}\right)=\int_{0}^{\infty} S^{s_{1}-1}\left(S+s_{2}\right)^{-1} e^{-s_{1} S} d S, \quad s_{1}=n+a, \quad s_{2}=\varepsilon / n \quad$ and $s_{3}=b+V-n \log \left(\min \left(\rho, x_{(1)}\right)\right)$.

Hence the Bayes estimator of $\theta$ under GEL is obtained as

$$
\hat{\theta}_{B E 1}=\left(\frac{J\left(s_{1}-p, s_{2}, s_{3}\right)}{J\left(s_{1}, s_{2}, s_{3}\right)}\right)^{-1 / p}
$$

and for particular $p=-1$ the Bayes estimator under GEL is the posterior mean and is given by

$$
\hat{\theta}_{B E 1}=\frac{J\left(s_{1}+1, s_{2}, s_{3}\right)}{J\left(s_{1}, s_{2}, s_{3}\right)}
$$

\section{Conclusion}

The shrinkage test-estimator $\hat{\theta}_{u S H}$ performs well with respect to the minimum risk estimator $Y_{1}$ for small $n$ and $k$, in the interval $0.50 \leq \delta \leq 1.50$. Similarly, the shrinkage test-estimator $\hat{\theta}_{u S H 1}$ is more efficient than $Y_{1}$ in the interval $0.50 \leq \delta \leq 1.75$, whereas the test-estimator $\hat{\theta}_{u S H} 2$ performs uniformly well for all considered values of parametric space. Therefore the shrinkage test-estimator $\hat{\theta}_{u S H}$ is preferable over others.

\section{References}

1. H. T. Davis and M. L. Feldstein, Biometrika 66, 299 (1979). doi:10.1093/biomet/66.2.299

2. J. Steindle, Random processes and the growth of firms: a study of the Pareto law (Hafner, New York, 1965). 
3. K. S. Lomax, Journal of the American Statistical Association 49, 847 (1954). doi: $10.2307 / 2281544$

4. C. M. Harris, Navel Research Logistics Quarterly 14, 219 (1967).doi:10.1002/nav.3800140207

5. E. C. Freiling, U.S. Naval Radiological Defense Laboratory (San Francisco, 1966).

6. C. M. Harris, Operations Research 16, 307 (1968). doi:10.1287/opre.16.2.307

7. D. Dyer, Canadian Journal of Statistics 9, 71 (1981). doi:10.2307/3315297

8. J. R. Thompson, Journal of American Statistical Association 63, 113 (1968). doi: $10.2307 / 2283832$

9. E. Harris and G. Shakarki, Journal of Chronical Disease 32, 233 (1979). doi:10.1016/0021-9681(79)90069-9

10. G. Tso, Proceeding of the ASA Section on Business and Commerce (1990).

11. R. J. Marshall, Journal of Applied Statistics 40, 283 (1991). doi:10.2307/2347593

12. D. A. Wooff, Journal of Royal Statistical Society B 47, 362 (1985).

13. B. N. Pandey and J. Singh, Journal of Indian Statistical Association 15, 141 (1977).

14. M. Pandey and S. K. Upadhyay, IEEE Transaction on Reliability, R-34, 491 (1985).

15. M. D. Conerly and J. M. Hardin, Communications in Statistics -Theory and Methods 20, 3591 (1991). doi:10.1080/03610929108830726

16. D. C. Singh, P. Singh and P. R. Singh, Microelectron Reliability 36 (3), 435 (1996). doi:10.1016/0026-2714(95)00002-X

17. G. Prakash and D. C. Singh, Austrian Journal of Statistics 35 (4), 463 (2006).

18. G. Prakash, D. C. Singh, and B. N. Pandey, Journal of Probability and Statistical Science 6 (2), 135 (2008).

19. H. R. Varian, In studies in Bayesian econometrics and statistics in honor of L. J. Savage, Eds S. E. Feinberge and A. Zellner (Amsterdam North Holland, 1975) p. 195.

20. R. Calabria and G.Pulcini, Communication in Statistics - Theory and Methods 25 (3), 585 (1996). 